\title{
Argumentation for Explicit Representation of Control within Enterprise Modelling and Integration
}

\author{
Bruno Vallespir, David Chen, and Guy Doumeingts
}

LAP/GRAI, University Bordeaux I - CNRS, France, vallespir@lap.u-bordeaux.fr

\begin{abstract}
The paper addresses the necessity and rationale to take enterprise control into account explicitly in order to ensure that the implemented architecture enables to get the level of performance required by the strategy of the company. To control a system that cannot be entirely formalised and modelled, it is necessary to provide a set of decision-makers with co-ordination links between them. Focusing on control means focusing on decision-making and on the role of man in modern industrial systems as well. Man-based decision-making oriented enterprise control is proposed as a complementary approach with respect to formalised views used in enterprise modelling and integration. The purpose of this paper is only to argue this proposition and not to give operational means to do so.
\end{abstract}

\section{INTRODUCTION}

Enterprise Engineering and Integration is a complex and interdisciplinary project in which not only technical aspects, but also human factors, control and decisional aspects must be considered (IFAC-IFIP, 1999). Within the current industrial challenge, control plays a major role for matching performances and then keeping a company competitive. However, the global control aspect is not well taken into account. From our point of view, most of existing approaches are information modelling oriented and the control is reduced to an information processing issue. This statement is not only ours, many others think that to match current industrial challenges, control needs

\footnotetext{
The original version of this chapter was revised: The copyright line was incorrect. This has been corrected. The Erratum to this chapter is available at DOI: 10.1007/978-0-387-35621-1_43 
to be in an equivalent position to information and communication (ICIMS, 1999).

Focusing on control means focusing on decision-making and on the role of man in industrial systems as well. It is necessary to avoid mechanistic approaches and to situate human decision into the system.

In this paper, firstly some theoretical arguments to justify the necessity of having a control view are formulated. Then basic concepts and principles to implement enterprise decision-making system are presented. The impacts of introducing human decision making to control industrial system are discussed.

\section{THEORETICAL ARGUMENTS AND BASIC CONCEPTS}

The traditional approach in system engineering coming from automation considers that a system has some significant states that can be formalised (i.e. explicitly identified and defined). Formalising these states enables to take them into account, i.e. to translate them into a model, which is used to control the system. To consider that the significant states can be formalised is worthy of remark. This assumption is the basis of automation. It leads the system to run "automatically" when it is in a formalised state or to be blocked or insensitive in other states. (In the largest meaning. An activity completely defined by a procedure, even if this activity is performed by a man, is considered here as to be "automatic".) However, this automatic control approach may be justified and used when (1) the product is cheap or the wasted raw material can be re-collected in case when the system is out of order; (2) the performances expected from the system are low so the decrease of the level of service is acceptable (waste of time for example); or (3) the risk to go out of the set of formalised states is low because the system is quasi-exhaustively modelled.

Many products we use everyday (domestic devices, PCs, etc.) belong to this class of systems. However, this is not the case for an industrial system: the system must not be damaged and cannot be reconstructed, the performances expected from the system do not allow any degradation of the result. Furthermore the system is weakly modelled, and this for two main reasons:

- It is generally possible to describe an operational process from a qualitative point of view but it is more difficult to quantify it. Very often, the lead-time is an average value with a large dispersion around it. This dispersion becomes larger when the number of human operators grows; 
- The number of situations and events influencing the system cannot be counted and exhaustively identified beforehand. A large and complex system such as an industrial system is rarely in a nominal mode. Very often, the model of the system is a description of an ideal process corresponding rarely to the reality.

How to control such a system? More precisely, how to control the system when it is in a significant but not formalised state? A principle from cybernetics gives a piece of solution: a system is really controlled only if the control system proposes at least as many states (control system variety) as the controlled system (controlled system variety). Because this variety does not exist $a$ priori, it is necessary to have inside the control system some variety generators, i.e. organs that are capable to react to a situation at the time the situation appears. The only organ known to be versatile and quick enough to play this role is the human being (Fig. 1).

The human being can play the role of variety generator if his or her freedom to make decision is high. This leads to a difficulty: a high degree of freedom can lead the decisionmaker to have a behaviour that is not compatible with the overall objectives of the system. Then, the decisional freedom needs to be limited in some way (framed). The problem grows when the number of decision-

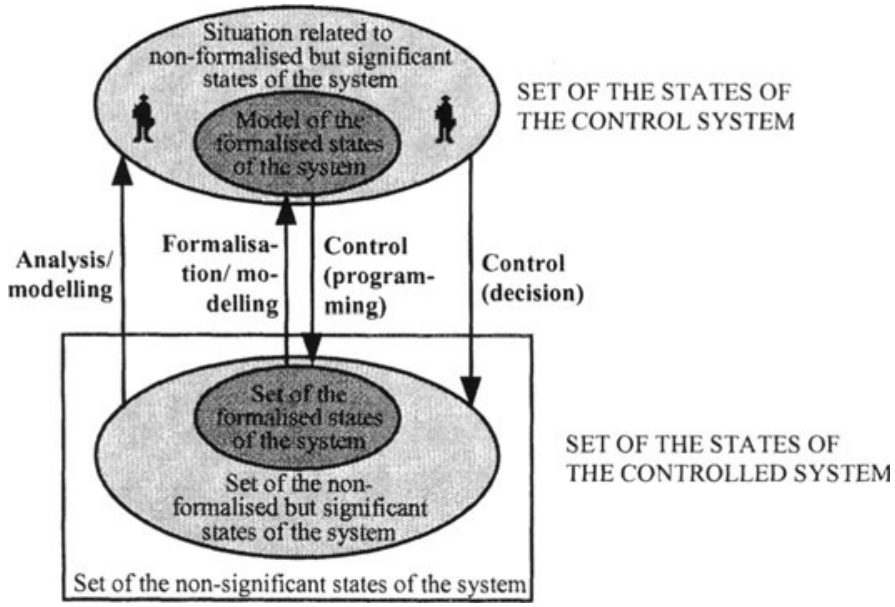

Figure 1: Increase of the control system variety by the presence of human beings makers grows.

There is a need to consistently frame a set of local decisional freedoms dealing with specific objectives. The solution is co-ordination in the meaning that decisional freedoms are defined and delimited by a co-ordinator module. Generally, the co-ordinator is in the same situation as the co-ordinated modules: it is co-ordinated too. Then the complex, multi-objectives systems, such as industrial systems, are controlled by a hierarchical, multi-level structure, which owns the important property of decentralisation of decision-making (Doumeingts et al., 1984). This aspect must not be confused with the organisation chart, which is concerned with tasks and responsibilities assignment. 
At the level of decision-making itself, we can consider that the main items to define a frame for the decision-making are (1) the valued objective or set of objectives the decision centre has to match and (2) the decision variables enabling the decision centre to know under what constraints it can act.

At the detailed level, decision-making may be considered to the search for a position in a space defined by decision variables and closed (limited) by constraints in order to process the received information and to match the objectives (Fig. 2).

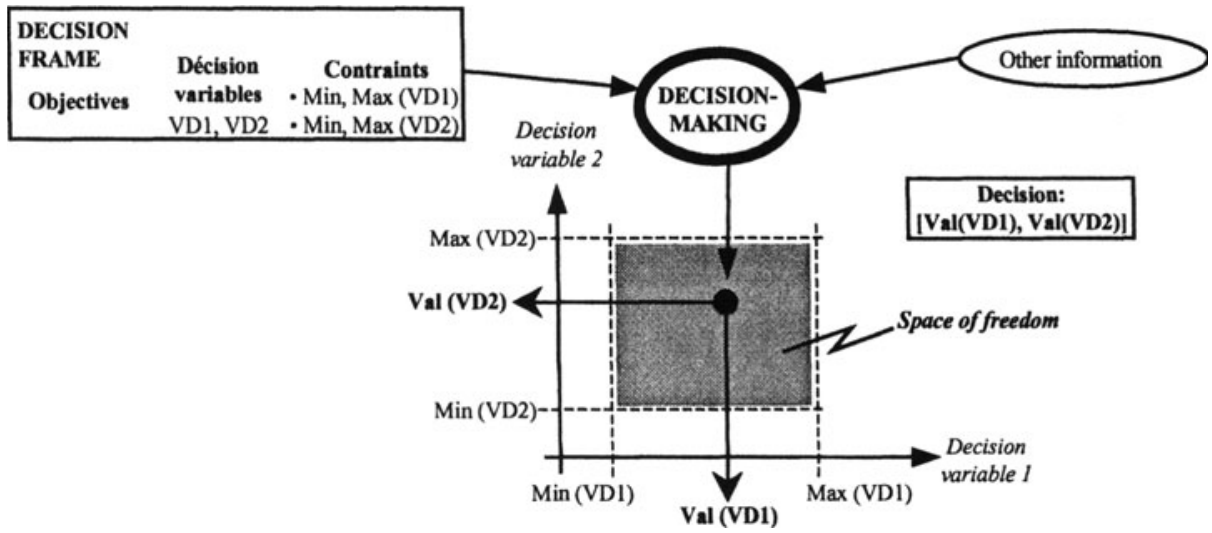

Figure 2: Principle of decision-making in conformity with a decision frame (here: only two decision variables are considered)

These concepts allow one to define a consistent decision-making environment rather than to show how to make an individual decision.

\section{IMPACT ON CONTROL AND PERFORMANCE}

An industrial system is an artefact, which must run in accordance with the objectives defined by the strategy of the company. This aspect has to be taken into account during the design phase and during the operating phase. Our interest for this section is the operating phase.

An industrial system has most of time a high degree of flexibility. This flexibility is absolutely necessary to match the "hard requirements" coming from the market. On the other hand, the potential risk is to use this flexibility in a bad way, leading to a mismatch with the objectives of the company. That is why control is today a key issue for reaching performances.

The main purpose of control is to ensure that operational activities run in a consistent way and that objectives coming from upper level (corporate 
strategy) are met. For that purpose, control must on one-hand co-ordinate tasks in relation to these objectives and to enable the deployment of these objectives into the structure and, on the other hand, follow up performances of operations in order to compare them to objectives. However, many industrial cases show that sometimes these follow-up activities are often not very usable or do not even exist at every level of the hierarchy and. Then, the main issue is to merge a top-down approach corresponding to the deployment of objectives and a bottom-up approach dealing with the follow-up of operations and aggregation of information (Fig. 3).

We find here the concept that a structure can really be controlled only if some feedback loops are implemented (in particular when the system is weakly modelled). The source of this feed-back is all the raw data coming from the operational processes and from the environment of the system. These raw data can be relevant for the lowest levels of control because these levels are close to the real system. However, they are not relevant for upper levels, for two reasons.

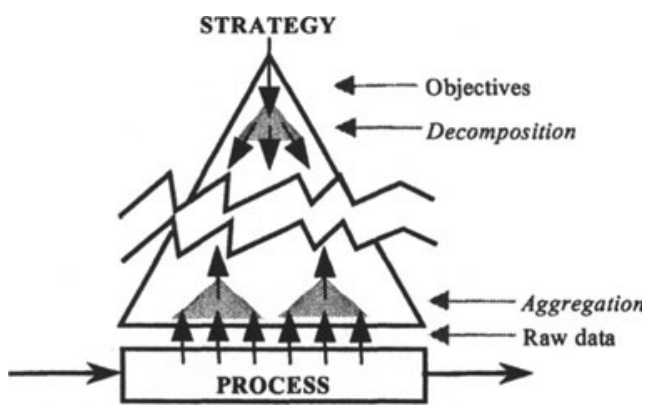

Figure 3: The issue of matching decomposition of objectives and aggregation of information

The first reason is the cognitive limitation of decision-makers. This limitation can be expressed in term of quantity of information. Beyond this limit, the decision-maker is submerged by the information: he or she is not able to interpret the supposedly useful information in order to make decision. The quantity of information is proportional to (1) the size of the domain in which the decision-maker is supposed to make decision and (2) the detail of the information handled by the decision-maker. Therefore information needs to be aggregated as it goes up to levels that have a broader and more conceptual view of the operational process in the structure, in order to provide the good granularity of information to decision-makers.

The second reason is related to the fact that decision is mainly made on a basis of objectives and

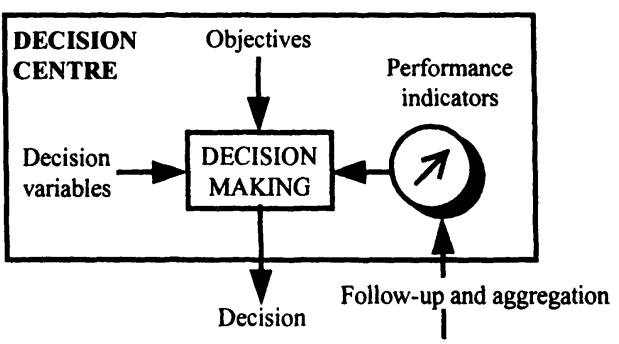

Figure 4: Decision centre and triplet \{Objectives, Decision Variables, Performance Indicators\} decision variables, so that information must be expressed in a way closely 
related to these two items (Doumeingts et al. 1992, 1995, 1998). This is the notion of performance indicator (Fig. 4).

Performance indicators must be consistent with objectives because it is necessary to compare the target performances (objectives) and performances reached (indicator) (observability). Performance indicators must be consistent with decision variables because these must have an effect on the performance concerned (controllability). Therefore the main issue is to ensure the internal consistency inside a decision centre in terms of the triplet of $o b$ jectives, decision variables and performance indicators. This consistency is ensured if the performance indicators allow the verification of the achievement of objectives, and if they are influenced by actions on decision variables.

\section{IMPACT ON DECISION-MAKING AND "MEN IN THE SYSTEM"}

Very often, objectives on quality, costs and lead times are enunciated simultaneously. The main problem is that all these objectives are looked for at the same time. Control is always multi-objective. That is why the problem of decision-making is of a tremendous importance because, most of time, decision-making is not reducible to the optimisation of one criterion. Two systems, with the same resources, products, etc. may have a very different level of performances in accordance with the quality of decisions made inside their control system. That is why it is very important for an industrial system to create the best environment for decision-making.
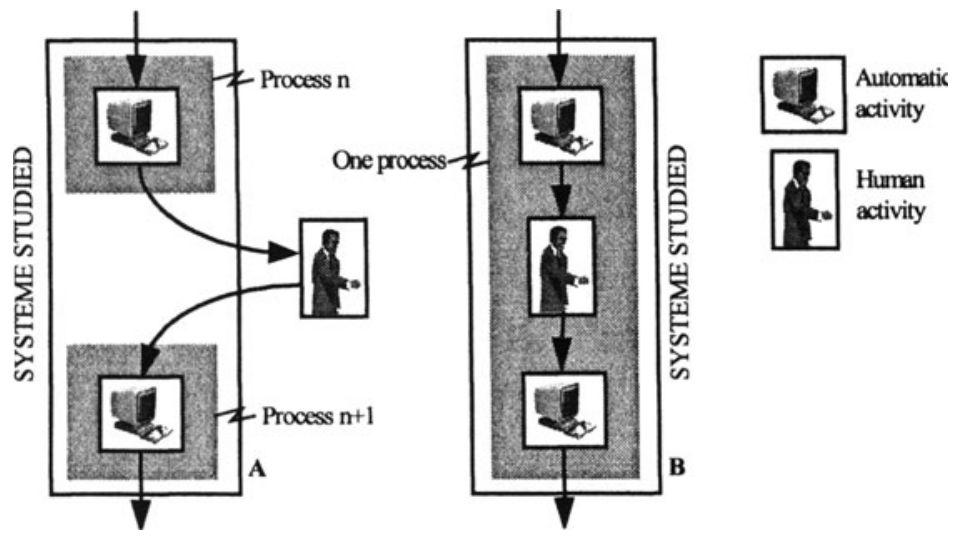

Figure 5: Presence of a men in processes: customer of the system and between two processes (A) or actor of the svstem and inside the process (B) 
Today, the essential role of man inside an industrial system is not to operate any more but to decide. Therefore to focus on decision-making in system engineering is also to focus on the position of men in the system.

The first effect of this consideration is to avoid any model where men would be considered outside the system (men = customers or service providers). One of the main concepts making Enterprise Modelling and Integration different to techno-centred approach such as automatic control, computer sciences, etc. is that men are completely part of the system and be involved in processes of the system (Fig. 5).

The techno-centred approach remains relevant when important automatic processes exist. However, this is not often the case in industrial systems for the reasons discussed previously.

The second effect is that is not reasonable to expect to model and formalise the internal running of decisional activities. In a modelling activity, it is attractive to try to do so because these activities belong to the studied process. This trend could be problematic because:

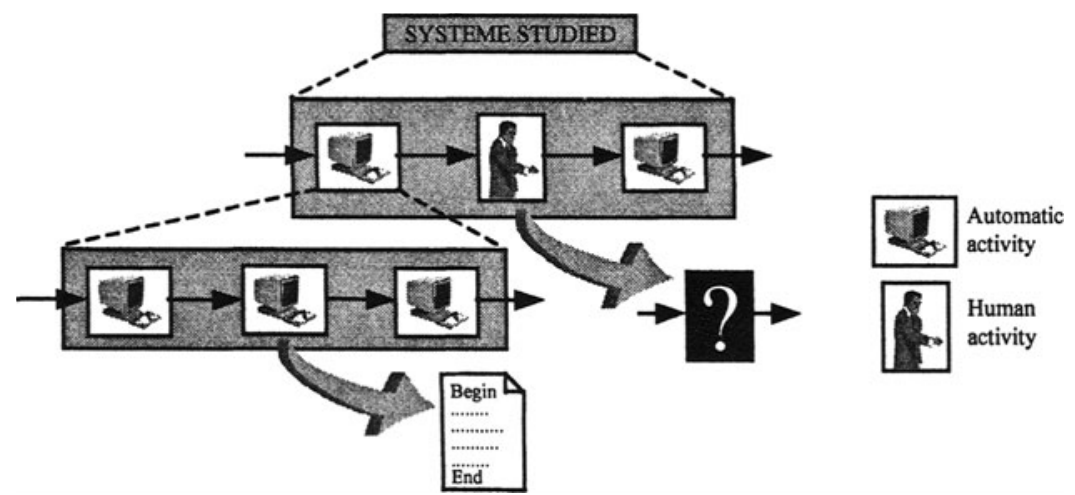

Figure 6: Modelling an "automatic" activity and a decisional activity

- Firstly, it will be generally disappointing because, despite the efforts to model these activities, men will present numerous behaviours outside the model. Therefore it is wiser to consider real decisional activities as black boxes whose the main inputs and outputs are known in order to situate them inside the process (Fig. 6);

- Secondly, it could be dangerous to formalise the activity in order to constraint men to a nominal behaviour. The capacity of the decisionmaker to generate a variety of decisions could be partially or completely inhibited by this constraint. This aspect is perfectly illustrated in some structures where the standardisation of activities has led to the loss of responsibility and imagination in abnormal situations. 


\section{CONCLUSIONS}

This paper has presented the necessity to take into account the control aspect in enterprise modelling and integration. This consideration has been argued and justified from a theoretical point of view. A set of basic concepts to implement decision centres with well defined triplets of information (Objectives, Decision Variable and Performance Indicators) to allow a systemwide consistent decision making have been proposed.

To take our statement into account, two levels of detail are concerned: (1) a local view enabling to define the environment of decision (information required, performance indicators, decision variables, etc.) and (2) a global view in order to define the architecture of decisions to implement coordination between decision centres and to ensure the deployment of corporate objectives throughout the industrial system.

\section{REFERENCES AND BIBLIOGRAPHY}

Doumeingts, G. (1984), Méthode GRAI: méthode de conception des systèmes en productique. Thèse d'état, University Bordeaux I.

Doumeingts, G. Vallespir, B. (1995), Les aspects humains dans la conception des systèmes de production - Proc. of 30th Congress of Société d'Ergonomie de Langue Française Biarritz, France.

Doumeingts, G., Vallespir, B. Chen, D. (1998), Decision modelling GRAI grid, Chapter in: Handbook on architecture for Information Systems, Peter Bernus, Kai Mertins, Gunter Schmidt, (Eds.). Springer-Verlag.

Doumeingts, G., Vallespir, B. Zanettin, M. Chen, D. (1992), GIM: GRAI Integrated Methodology for designing CIM systems, GRAI/LAP, University Bordeaux I, version 1.0.

ICIMS (2000), ICIMS-NOE Scientific meeting, Brussels, Belgium, November 24, 1999, in ICIMS NEWS, March.

IFAC-IFIP Task Force (1999), GERAM: Generalized Enterprise Reference Architecture and Methodology, Version 1.6.2, Annex A in IS 15704, Requirements for Enterprise Reference Architecture and Methodologies, ISO TC 184/SC5/WG1. 